Draft VERSION July 27, 2021

Preprint typeset using $\mathrm{L}^{A} \mathrm{~T}_{\mathrm{E}} \mathrm{X}$ style emulateapj v. 01/23/15

\title{
SN 2009ip: CONSTRAINING THE LATEST EXPLOSION PROPERTIES BY ITS LATE-PHASE LIGHT CURVE
}

\author{
TAKASHI J. MORIYA \\ Argelander Institute for Astronomy, University of Bonn, Auf dem Hügel 71, 53121 Bonn, Germany \\ Draft version July 27, 2021
}

\begin{abstract}
We constrain the explosion and circumstellar properties at the 2012b event of SN 2009ip based on its late-phase bolometric light curve recently reported. The explosion energy and ejected mass at the $2012 \mathrm{~b}$ event are estimated as $0.01 M_{\odot}$ and $2 \times 10^{49} \mathrm{erg}$, respectively. The circumstellar medium is assumed to have two components: an inner shell and an outer wind. The inner shell which is likely created at the $2012 \mathrm{a}$ event has $0.2 M_{\odot}$. The outer wind is created by the wind mass loss before the 2012a mass ejection, and the progenitor is estimated to have had the mass-loss rate about $0.1 M_{\odot} \mathrm{yr}^{-1}$ with the wind velocity $550 \mathrm{~km} \mathrm{~s}^{-1}$ before the 2012a event. The estimated explosion energy and ejected mass indicate that the $2012 \mathrm{~b}$ event is not caused by a regular supernova.
\end{abstract}

Keywords: supernovae: individual (SN 2009ip) — stars: mass-loss — stars: massive

\section{INTRODUCTION}

It is widely believed that the efficient conversion of kinetic energy to radiation results in luminous transients. Type IIn supernovae (SNe), which sometimes even become superluminous (e.g., Gal-Yam 2012), are largely powered by the interaction between SN ejecta and circumstellar media (CSM) (e.g., Moriya et al. 2014). There also exist bright transients called 'SN impostors' which are likely brightened by the collision of the material ejected from the progenitor intermittently (e.g., Van Dyk et al. 2000). SN impostors are not caused by the final SN explosions of the progenitors, but they sometimes become as bright as SNe. Type IIn SNe and SN impostors are related to the unsolved problems in stellar mass loss, and it is important to understand their origins (e.g., Smith 2014; Langer 2012). They are also suggested to be an important high-energy cosmic-ray producers (Murase et al. 2011, 2014). In addition, they can play a role as a distance ladder (Potashov et al. 2013) and can also be an important probe of the early Universe (e.g., Cooke et al. 2009; Tanaka et al. 2012).

SN 2009ip is one of the most studied transients powered by the interaction due to its activeness which kept us surprised since 2009. The progenitor of SN 2009ip got bright and was assigned a SN name in August 2009 (Maza et al. 2009). However, the subsequent observations revealed that it was not a genuine SN and the progenitor remained at the location (Smith et al. 2010; Foley et al. 2011). The progenitor mass is estimated to be above $\sim 60 M_{\odot}$, and the observed brightening is considered to be a SN impostor from a luminous blue variable star (Smith et al. 2010; Foley et al. 2011). The progenitor experienced several rebrightening since 2009 (Pastorello et al. 2013).

SN 2009ip showed drastic changes in 2012 (Fig. 1). In August 2012, SN 2009ip started to be bright again and faded temporarily after about 40 days (the 2012a event, see Fig. 1). Then, it became bright again and reached the peak luminosity of $8 \times 10^{42} \mathrm{erg} \mathrm{s}^{-1}$ (Pastorello et al. 2013), which is comparable to those observed in SNe

moriyatk@astro.uni-bonn.de (the 2012b event, Fig. 1). The observations during and after the 2012 events of SN 2009ip are reported by many authors (Prieto et al. 2013; Pastorello et al. 2013; Fraser et al. 2013, 2015; Margutti et al. 2014; Ofek et al. 2013a; Smith et al. 2013, 2014; Mauerhan et al. 2013, 2014; Levesque et al. 2014; Graham et al. 2014; Martin et al. 2015; Fox et al. 2015).

The origin of the final luminosity increase observed so far (the 2012b event) has been largely debated. The peak luminosity which is comparable to those of SNe and the broad spectral lines led to the suggestion that the $2012 \mathrm{~b}$ event is triggered by the final SN explosion of the progenitor (e.g., Mauerhan et al. 2013; Smith et al. 2014; Baklanov et al. 2013; Ouyed et al. 2013). The 2012a event, which is similar to the precursor of SN 2010mc (Ofek et al. 2013b), is linked to the pre-SN mass ejection probably caused by the violent late-phase nuclear burning (e.g., Quataert \& Shiode 2012, see also Moriya 2014a). On the other hand, it is also suggested that a SN event may not be required to explain the large luminosity observed in the $2012 \mathrm{~b}$ event because of the efficient conversion from the kinetic energy to radiation. Thus, the 2012 b event may not be caused by the final SN explosion of the progenitor (e.g., Pastorello et al. 2013; Fraser et al. 2013, 2015; Margutti et al. 2014). For example, Soker \& Kashi (2013); Kashi et al. (2013); Tsebrenko \& Soker (2013) related the 2012 events to the merger of massive stars.

The aim of this Letter is to reveal the unresolved origin of the enigmatic $2012 \mathrm{~b}$ event by modeling its late-phase bolometric light curve (LC) until about 750 days after the 2012 b event recently reported by Fraser et al. (2015). We constrain the explosion and circumstellar properties at the 2012b event by using an analytic bolometric LC model developed by Moriya et al. (2013b). We begin this Letter by briefly summarizing our LC model in the next section.

\section{LIGHT-CURVE MODEL}

\subsection{Assumptions}

We assume a progenitor system schematically shown in Fig. 2 led to the 2012b and later event of SN 2009ip. 


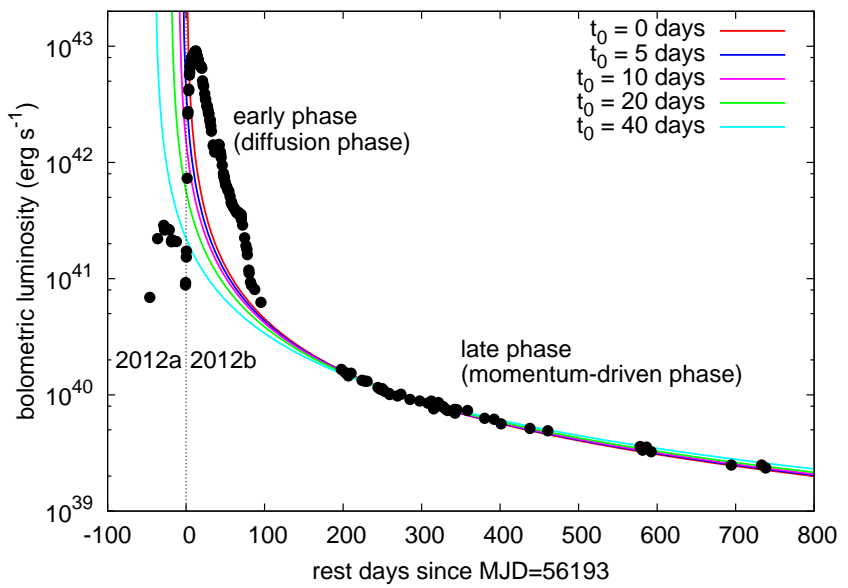

Figure 1. Bolometric LC of SN 2009ip in 2012 and later shown in Fraser et al. (2015). The origin of time is set at the beginning of the $2012 \mathrm{~b}$ event. We also show the results of the LC fitting with the function $L=L_{1}\left(t+t_{0}\right)^{-1.5}$ after 150 days since the beginning of the $2012 \mathrm{~b}$ event.

The event is assumed to be caused by an ejection of the mass $M_{\mathrm{ex}}$ with the kinetic energy $E_{\mathrm{ex}}$ in a dense CSM. The dense CSM are assumed to have two components: an inner shell and an outer regular wind. The inner shell is presumed to be created by the mass ejection during the 2012 a event with the mass-loss rate $\dot{M}_{\text {ej }}$, and it is assumed to have the mass $M_{\mathrm{sh}}$. We note that this kind of shells may also be formed by the confinement of the stellar wind (Mackey et al. 2014). The outer wind structure is assumed to be created with wind mass loss with the mass-loss rate $\dot{M}_{\mathrm{s}}$ and velocity $v_{\mathrm{w}}$. Although the observed luminosity fluctuations before the 2012a event indicate that the CSM may not be smooth, we assume that the overall density structure is approximately proportional to $r^{-2}$. Based on the observations of SN 2009ip in 2009, we assume that $v_{\mathrm{w}}=550 \mathrm{~km} \mathrm{~s}^{-1}$ (Smith et al. 2010; Foley et al. 2011). The inner and outer components are separated at $R_{\text {sh }}$.

We assume that the inner shell is responsible for the rise and decline observed until $\sim 100$ days since the $2012 \mathrm{~b}$ event ('early phase', Section 2.2). Then, a shock containing both $M_{\mathrm{ex}}$ and $M_{\mathrm{sh}}$ is assumed to propagate in the outer wind. The shock is assumed to be thin due to the efficient radiative cooling. The continuous interaction between the shock and the outer wind is assumed to be responsible for the later ( $\gtrsim 200$ days) LC reported by Fraser et al. (2015) ('late phase', Section 2.3).

Finally, we note that the spherical symmetry is assumed in our analytic model. Some observations of the 2012 b event are suggested to indicate that the CSM is aspherical (e.g., Levesque et al. 2014; Mauerhan et al. 2014; Graham et al. 2014). However, the mass-loss rates obtained by assuming the spherical symmetry are still likely to be good estimates for the actual mass-loss rates in the aspherical systems (Moriya et al. 2014). In addition, the conversion efficiency discussed below also partly contains the effect of the asphericity (Moriya et al. 2014).

\subsection{Early phase (diffusion phase)}

We assume that the early phase is caused by the diffusion in the inner shell (see also Margutti et al. 2014; Ofek et al. 2013a). If we assume that the shell has an aver-

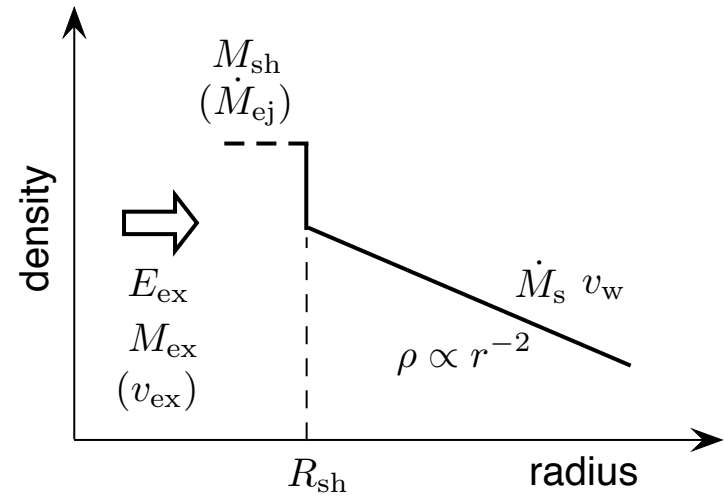

Figure 2. Schematic illustration of the explosion and circumstellar properties assumed in our model. The ejecta with the kinetic energy $E_{\text {ex }}$ and the mass $M_{\text {ex }}$ exploded at the $2012 \mathrm{~b}$ event first collides with the inner shell with $M_{\text {sh }}$ created at the 2012a event with the mass-loss rate $\dot{M}_{\text {ej }}$. Then, the thin shock containing both $M_{\mathrm{ex}}$ and $M_{\mathrm{sh}}$ interacts with the outer wind created by the progenitor prior to the 2012 a event with the mass-loss rate $\dot{M}_{\mathrm{s}}$ and velocity $v_{\mathrm{w}}$.

age density $\bar{\rho}$, the diffusion time $t_{\text {diff }}$ in the shell, which corresponds to the rise time of the LC, is expressed as

$$
t_{\mathrm{diff}} \simeq \frac{\kappa \bar{\rho} R_{\mathrm{sh}}^{2}}{c},
$$

where $\kappa$ is opacity and $c$ is the speed of light. We assume $\kappa=0.34 \mathrm{~cm}^{2} \mathrm{~g}^{-1}$ in this study. Using the diffusion time, $M_{\text {sh }}$ can be estimated as

$$
M_{\mathrm{sh}} \simeq \frac{4}{3} \pi \bar{\rho} R_{\mathrm{sh}}^{3}=\frac{4 \pi c t_{\mathrm{diff}} R_{\mathrm{sh}}}{3 \kappa} .
$$

As the rise time and the $e$-folding time of the later luminosity decline are both about 14 days (e.g., Pastorello et al. 2013; Moriya et al. 2014), a shock breakout is likely occurred in the shell (Margutti et al. 2014; Ofek et al. 2013a). This indicates that the entire shell is shocked at the LC peak. Thus, we presume that the blackbody radius at the LC peak of the $2012 \mathrm{~b}$ event corresponds to $R_{\text {sh }}$ and we set $R_{\text {sh }} \simeq 10^{15} \mathrm{~cm}$ (Margutti et al. 2014). Assuming $t_{\text {diff }} \simeq 14$ days and $R_{\text {sh }} \simeq 10^{15} \mathrm{~cm}$, we obtain $M_{\mathrm{sh}} \simeq 0.22 M_{\odot}$. The estimated mass is consistent with those obtained in the previous studies $\left(\sim 0.1 M_{\odot}\right.$, e.g., Fraser et al. 2013; Margutti et al. 2014). If the shell is ejected during the 2012a event which lasted for $\sim 40$ days, the mass-loss rate during the 2012 a event becomes $\dot{M}_{\mathrm{ej}} \simeq 2 M_{\odot} \mathrm{yr}^{-1}$.

The explosion properties $\left(E_{\mathrm{ex}}\right.$ and $\left.M_{\mathrm{ex}}\right)$ can be related to the total radiation energy emitted during the $2012 \mathrm{~b}$ event. The conservation of momentum and energy results in (e.g., Moriya et al. 2013a)

$$
E_{\mathrm{rad}}=\frac{M_{\mathrm{sh}}}{M_{\mathrm{ex}}+M_{\mathrm{sh}}} E_{\mathrm{ex}} .
$$

The total energy during the $2012 \mathrm{~b}$ event is estimated to be $E_{\text {rad }} \simeq 2 \times 10^{49}$ erg (Fraser et al. 2013; Margutti et al. 2014).

\subsection{Late phase (momentum-driven phase)}

Fraser et al. (2015) recently reported the late phase LCs until about 750 days after the $2012 \mathrm{~b}$ event and constructed a bolometric LC (Fig. 1). The late bolometric 
LC is shown to evolve with a power law $L=L_{1} t^{-\alpha}$, where $t$ is time since the explosion. Fraser et al. (2015) fitted the power-law function by setting $t=0$ at the beginning of the 2012a event and obtained $\alpha=1.74$ as the best fit parameter. However, we here assume that the inner explosion causing the $2012 \mathrm{~b}$ event occurred after the 2012a event. If we set $t=0$ at MJD $=56193$ when the $2012 \mathrm{~b}$ event began, we obtain $\alpha=1.44$ as the best fit parameter.

What is surprising is that $\alpha$ is near 1.5 and it is significantly larger than 1.0. If the interaction between SN ejecta and the dense wind is still ongoing, $\alpha$ is expected to be significantly below 1.0 when the wind is almost steady because of the continuous momentum injection from the SN ejecta (e.g., Moriya et al. 2013b; Ofek et al. 2014). For example, in the case of Type IIn SN 2010jl, the bolometric LC follows a power law with $\alpha<1$ even at around 600 days after the explosion (e.g., Maeda et al. 2013), although there exist some arguments for the LC interpretation (Fransson et al. 2014).

The large $\alpha$ near 1.5 in the LC after about 200 days since the 2012b explosion indicates that the shock proceeding in the wind is already in the momentum-driven phase at about 200 days. The momentum-driven phase (also called 'snow-plow phase') is the phase when the momentum injection to the shock has already terminated and the shock moves only with the momentum injected to the shock previously (see, e.g., Svirski et al. 2012; Ofek et al. 2014; Moriya 2014b). Moriya et al. (2013b) obtained the luminosity evolution during the momentum-driven phase in the steady wind as

$$
L=\frac{\epsilon}{2} \frac{\dot{M}_{\mathrm{s}}}{v_{\mathrm{w}}}\left(\frac{2 E_{\mathrm{m}}}{M_{\mathrm{m}}}\right)^{\frac{3}{2}}\left[1+2 \frac{\dot{M}_{\mathrm{s}}}{v_{\mathrm{w}}}\left(\frac{2 E_{\mathrm{m}}}{M_{\mathrm{m}}^{3}}\right)^{\frac{1}{2}} t\right]^{-\frac{3}{2}},
$$

where $\epsilon$ is the conversion efficiency from the kinetic energy to radiation, and $E_{\mathrm{m}}$ and $M_{\mathrm{m}}$ is the energy and mass released inside the wind, respectively. In the system we are interested in (Fig. 2), the shock propagates in the outer wind component after it has passed through the inner shell. Thus, we can set

$$
M_{\mathrm{m}}=M_{\mathrm{ex}}+M_{\mathrm{sh}},
$$

from the conservation of mass, and

$$
M_{\mathrm{m}} E_{\mathrm{m}}=M_{\mathrm{ex}} E_{\mathrm{ex}},
$$

from the conservation of momentum.

The luminosity evolution in the momentum-driven phase (Eq. 4) can be separated into two parts. At first, when $2\left(\dot{M}_{\mathrm{s}} / v_{\mathrm{w}}\right)\left(2 E_{\mathrm{m}} / M_{\mathrm{m}}^{3}\right)^{1 / 2} t \lesssim 1$, the luminosity is constant. This is because the wind mass swept by the shock is much smaller than the initial injected mass $\left(M_{\mathrm{m}}\right)$ and the shock freely expands with a constant velocity. Then, when

$$
2 \frac{\dot{M}_{\mathrm{s}}}{v_{\mathrm{w}}}\left(\frac{2 E_{\mathrm{m}}}{M_{\mathrm{m}}^{3}}\right)^{\frac{1}{2}} t \gtrsim 1
$$

starts to hold after the shock has swept a large amount of the wind (see Moriya 2014b for detailed discussion on this condition), the luminosity evolves as $L=L_{1} t^{-1.5}$, where

$$
L_{1}=2^{-\frac{7}{4}} \epsilon\left(\frac{\dot{M}_{\mathrm{s}}}{v_{\mathrm{w}}}\right)^{-\frac{1}{2}} E_{\mathrm{m}}^{\frac{3}{4}} M_{\mathrm{m}}^{\frac{3}{4}} .
$$

Using Eq. (8), the condition (7) can be used to constrain $M_{\mathrm{m}}$, i.e.,

$$
M_{\mathrm{m}} \lesssim 2^{\frac{4}{3}} \epsilon^{-\frac{1}{3}} L_{1}^{\frac{1}{3}}\left(\frac{\dot{M}_{\mathrm{s}}}{v_{\mathrm{w}}}\right)^{\frac{2}{3}} t^{\frac{1}{2}}
$$

The late-phase bolometric LC of SN 2009ip indicates that the condition (9) is satisfied at least about 200 days after the explosion.

In Fig. 1, we show the results of fitting of the function $L=L_{1}\left(t+t_{0}\right)^{-1.5}$ to the bolometric LC after 150 days since the beginning of the $2012 \mathrm{~b}$ event with several different explosion times $\left(t_{0}\right)$ relative to the beginning of the $2012 \mathrm{~b}$ event ( $\mathrm{MJD}=56193$ ). Although $\alpha=1.5$ is fixed in the fitting, decent fits to the bolometric LC are obtained. The best $L_{1}$ is found as $1.15 \times 10^{51}\left(t_{0}=0\right.$ days $)$, $1.18 \times 10^{51}\left(t_{0}=5\right.$ days $), 1.22 \times 10^{51}\left(t_{0}=10\right.$ days $)$, $1.29 \times 10^{51}\left(t_{0}=20\right.$ days $)$, and $1.42 \times 10^{51}\left(t_{0}=40\right.$ days $)$ in the cgs unit. Since $L_{1}$ does not depend strongly on $t_{0}$, we use $L_{1}=1.15 \times 10^{51} \mathrm{cgs}\left(t_{0}=0\right.$ days $)$ as a representative value in the following discussion.

Finally, the mass ejected at the inner explosion $\left(M_{\mathrm{ex}}\right)$ can be expressed as

$M_{\mathrm{ex}}=-\frac{M_{\mathrm{sh}}}{2}+\frac{1}{2}\left[M_{\mathrm{sh}}^{2}+2^{\frac{13}{3}} \epsilon^{-\frac{4}{3}} L_{1}^{\frac{4}{3}}\left(\frac{\dot{M}_{\mathrm{s}}}{v_{\mathrm{w}}}\right)^{\frac{2}{3}} M_{\mathrm{sh}} E_{\mathrm{rad}}^{-1}\right]^{\frac{1}{2}}$,

using Eqs. (3), (5), (6), and (8).

\section{EXPLOSION AND CIRCUMSTELLAR PROPERTIES AT THE 2012b EVENT OF SN 2009ip}

We now look into the explosion and circumstellar properties of the final explosive event observed in SN 2009ip so far. We have already constrained the inner shell mass in the previous section $\left(M_{\mathrm{sh}} \simeq 0.22 M_{\odot}\right)$. The wind velocity is fixed to $v_{\mathrm{w}}=550 \mathrm{~km} \mathrm{~s}^{-1}$. We also set $\epsilon=0.3$, which is typically found in Type IIn SN studies (e.g., van Marle et al. 2010; Fransson et al. 2014). The conversion efficiency is related to the physical properties of radiative shocks, and it is not likely to be altered by the origins of the explosions inside. Thus, we use a typical value found in the SN studies here. The conversion efficiency can be reduced by, e.g., multi-dimensional motions and asphericity (e.g., Moriya et al. 2013a). The observational information we have is $E_{\mathrm{rad}} \simeq 2 \times 10^{49} \mathrm{erg}$ and $L_{1} \simeq$ $1.15 \times 10^{51} \mathrm{cgs}$. We first assume several mass-loss rates for the outer wind $\left(\dot{M}_{\mathrm{s}}=10^{-1}, 10^{-2}, 10^{-3} M_{\odot} \mathrm{yr}^{-1}\right)$ and give constraints on the other parameters for the assumed mass-loss rates.

The explosion properties at the $2012 \mathrm{~b}$ event can be easily constrained with the formulae derived in Section 2. First, the ejected mass $M_{\text {ex }}$ can be constrained with Eq. (10). Then, the explosion energy $E_{\text {ex }}$ can be estimated with Eq. (3) assuming the obtained $M_{\text {ex }}$ and $M_{\text {sh }}$. Finally, we need to check if the condition (9) holds for the estimated parameters for consistency.

Table 1 summarizes the estimated parameters for SN 2009ip. We can first find that $M_{\text {ex }}$ is much smaller than $M_{\mathrm{sh}}$. This means that most of the kinetic energy released at the $2012 \mathrm{~b}$ event is converted to radiation energy (Eq. 3). In other words, Eq. (3) indicates that $E_{\mathrm{rad}} \simeq E_{\mathrm{ex}}$, and only small amount of the released ki- 
Table 1

Estimated explosion and circumstellar properties at the $2012 \mathrm{~b}$ event

\begin{tabular}{cccccc}
\hline$\dot{M}_{\mathrm{s}}{ }^{\mathrm{a}}$ & $E_{\text {ex }}$ & $M_{\text {ex }}$ & $M_{\text {sh }}$ & $E_{\mathrm{m}}$ & $v_{\text {ex }}$ \\
$M_{\odot} \mathrm{yr}^{-1}$ & $10^{49} \mathrm{erg}$ & $M_{\odot}$ & $M_{\odot}$ & $10^{47} \mathrm{erg}$ & $10^{4} \mathrm{~km} \mathrm{~s}^{-1}$ \\
\hline $10^{-1}$ & 2.1 & $1.1 \times 10^{-2}$ & 0.22 & 10 & 0.98 \\
$10^{-2}$ & 2.02 & $2.5 \times 10^{-3}$ & 0.22 & 2.2 & 2.0 \\
$10^{-3}$ & 2.005 & $5.3 \times 10^{-4}$ & 0.22 & 0.48 & 4.3 \\
\hline
\end{tabular}

${ }^{\mathrm{a}} v_{\mathrm{w}}=550 \mathrm{~km} \mathrm{~s}^{-1}$

netic energy is available for the late phase. The remaining kinetic energy $\left(E_{\mathrm{m}}\right)$ is only below $10 \%$ of $E_{\text {ex }}$ (Table 1). The total amount of energy radiated after 100 days obtained by assuming $L=L_{1} t^{-1.5}$ is $7.8 \times 10^{47} \mathrm{erg}$, and only the $10^{-1} M_{\odot} \mathrm{yr}^{-1}$ model is consistent with the total radiated energy.

The fact that the late-phase bolometric LC roughly follows $\propto t^{-1.5}$ gives the constraint (9). Assuming $t \simeq$ 200 days in Eq. (9), we obtain the following constraint:

$$
M_{\mathrm{ex}}+M_{\mathrm{sh}} \lesssim \begin{cases}0.2 M_{\odot} & \left(\dot{M}_{\mathrm{s}}=10^{-1} M_{\odot} \mathrm{yr}^{-1}\right), \\ 0.04 M_{\odot} & \left(\dot{M}_{\mathrm{s}}=10^{-2} M_{\odot} \mathrm{yr}^{-1}\right),(11) \\ 0.009 M_{\odot}\left(\dot{M}_{\mathrm{s}}=10^{-3} M_{\odot} \mathrm{yr}^{-1}\right) .\end{cases}
$$

Because $M_{\mathrm{sh}}$ is estimated to be $0.22 M_{\odot}$ and $M_{\mathrm{sh}} \gg$ $M_{\text {ex }}$, this constraint also indicates $\dot{M}_{\mathrm{s}}$ is around $10^{-1} M_{\odot} \mathrm{yr}^{-1}$. The estimated mass-loss rate is consistent with those estimated by the $\mathrm{H} \alpha$ luminosity (Fraser et al. 2013; Ofek et al. 2013a), while it is lower than that estimated by Ofek et al. (2013a) based on the multiwavelength observations. Combining above all, we suggest $M_{\mathrm{ex}} \simeq 0.011 M_{\odot}$ and $E_{\mathrm{ex}} \simeq 2.1 \times 10^{49} \mathrm{erg}$ as the explosion properties at the $2012 \mathrm{~b}$ event.

The estimated $E_{\text {ex }}$ and $M_{\text {ex }}$ with $\dot{M}_{\mathrm{s}}=10^{-1} M_{\odot} \mathrm{yr}^{-1}$ are also consistent with the interpretation that the shock breakout occurred in the inner shell at the $2012 \mathrm{~b}$ event (Section 2.2). The explosion velocity $v_{\mathrm{ex}} \equiv \sqrt{2 E_{\mathrm{ex}} / M_{\mathrm{ex}}}$ is shown in Table 1. The explosion velocity indicates that the shock breakout occurs where the optical depth is $\sim c / v_{\text {ex }} \simeq 30$ (e.g., Weaver 1976). If we use $\bar{\rho} \simeq$ $10^{-13} \mathrm{~g} \mathrm{~cm}^{-3}$ estimated by Eq. (1), the shock breakout occurs at $\simeq 2 \times 10^{14} \mathrm{~cm}$. This radius is near $\simeq 4-$ $5 \times 10^{14} \mathrm{~cm}$, which is the smallest photospheric radius observed at the beginning of the $2012 \mathrm{~b}$ event (Margutti et al. 2014).

The estimated average ejecta velocity of $v_{\mathrm{ex}} \simeq$ $10^{4} \mathrm{~km} \mathrm{~s}^{-1}$ is also consistent with the broad spectra observed in the 2012b event of SN 2009ip indicating the ejecta velocity of $\simeq 10^{4} \mathrm{~km} \mathrm{~s}^{-1}$ (e.g., Fraser et al. 2013). Pastorello et al. (2013) reported similarly broad spectral lines in SN 2009ip before the 2012 events, and this kind of broad lines are known to be associated with non-SN events.

The estimated explosion energy and mass at the 2012b event are not those of regular SNe which typically have $\sim$ $10^{51} \mathrm{erg}$ and $\sim 1-10 M_{\odot}$. In particular, the progenitor of SN 2009ip is likely heavier than $60 M_{\odot}$ (Smith et al. 2010; Foley et al. 2011), and the estimated small ejecta mass is inconsistent with its successful SN explosion. Even if the ejecta has $\sim 10^{51} \mathrm{erg}$ and only the ejecta within a certain direction interacted with the dense CSM to emit only $1 \%$ of the kinetic energy $\left(\sim 10^{49}\right.$ erg, e.g., Smith et al. 2014), the estimated ejecta mass is much smaller than $1 \%$ of the progenitor mass. Thus, the explosion occurred inside at the $2012 \mathrm{~b}$ event is not likely related to a regular SN. However, some SNe may have properties similar to those estimated here, and we cannot conclude for sure if the core collapse of the progenitor occurred or not. For example, a SN with large fallback can have a small amount of ejecta with a small kinetic energy (e.g., Moriya et al. 2010). Low energy and small mass ejection is also predicted to be caused by failed SNe (Nadezhin 1980; Lovegrove \& Woosley 2013). Massive star mergers may also have a similar fast mass ejection (e.g., Soker \& Kashi 2013).

\section{CONCLUSIONS}

We have estimated the explosion and circumstellar properties at the 2012b event of SN 2009ip based on the late-phase bolometric LC recently reported by Fraser et al. (2015). The bolometric LC roughly follows $L \propto t^{-1.5}$ at least from about 200 days after the $2012 \mathrm{~b}$ event (Fig. 1), and it indicates that the shock is already at the momentum-driven phase without any momentuminjection from inside at that time. Thus, we use an analytic bolometric LC model for the momentum-driven phase to estimate the explosion and circumstellar properties. We assume that an explosion occurred inside the circumstellar medium with two components: an inner shell and an outer wind (Fig. 2). The inner shell is supposed to be created during the 2012a event of SN 2009ip, while the outer wind is made by the wind mass loss of the progenitor prior to the $2012 \mathrm{a}$ event.

Combining all the bolometric LC information available after the $2012 \mathrm{~b}$ event, we suggest that an explosion with the energy $2.1 \times 10^{49} \mathrm{erg}$ and the mass $0.011 M_{\odot}$ occurred at the 2012b event of SN 2009ip. The ejecta first collided to the inner shell whose mass is estimated to be $0.22 M_{\odot}$. The collision between the ejecta and the inner shell is responsible for the early LC during the $2012 \mathrm{~b}$ event which is dominated by the photon diffusion after the shock breakout in the shell. The thin shock made by the efficient cooling, which contains the mass of both the ejecta and the inner shell, continues to travel in the outer wind, powering the late-phase LC of SN 2009ip. The total radiated energy and the fact that the bolometric LC is already at the momentum-driven phase with $L \propto t^{-1.5}$ at about 200 days after the explosion indicate that the mass-loss rate of the progenitor prior to the $2012 \mathrm{a}$ event is about $0.1 M_{\odot} \mathrm{yr}^{-1}$ with the wind velocity $550 \mathrm{~km} \mathrm{~s}^{-1}$.

The estimated explosion properties are not those of regular SNe. Thus, the explosion at the $2012 \mathrm{~b}$ event is not related to a regular SN. It is likely to be a non-SN explosive event or a peculiar SN like those accompanied with large fallback or caused by failed SN explosions.

I would like to thank the referee for the constructive comments and Morgan Fraser for sending me the electric data of the bolometric LC of SN 2009ip. The author is supported by Japan Society for the Promotion of Science Postdoctoral Fellowships for Research Abroad (26.51).

\section{REFERENCES}

Baklanov, P. V., Blinnikov, S. I., Potashov, M. S., \& Dolgov, A. D. 2013, Journal of Experimental and Theoretical Physics Letters, 98, 432 
Cooke, J., Sullivan, M., Barton, E. J., et al. 2009, Nature, 460, 237

Foley, R. J., Berger, E., Fox, O., et al. 2011, ApJ, 732, 32

Fox, O. D., Silverman, J. M., Filippenko, A. V., et al. 2015, MNRAS, 447, 772

Fransson, C., Ergon, M., Challis, P. J., et al. 2014, ApJ, 797, 118

Fraser, M., Inserra, C., Jerkstrand, A., et al. 2013, MNRAS, 433, 1312

Fraser, M., Kotak, R., Pastorello, A., et al. 2015 , arXiv:1502.06033v1

Gal-Yam, A. 2012, Science, 337, 927

Graham, M. L., Sand, D. J., Valenti, S., et al. 2014, ApJ, 787, 163

Kashi, A., Soker, N., \& Moskovitz, N. 2013, MNRAS, 436, 2484

Langer, N. 2012, ARA\&A, 50, 107

Levesque, E. M., Stringfellow, G. S., Ginsburg, A. G., Bally, J., \& Keeney, B. A. 2014, AJ, 147, 23

Lovegrove, E., \& Woosley, S. E. 2013, ApJ, 769, 109

Mackey, J., Mohamed, S., Gvaramadze, V. V., et al. 2014, Nature, 512, 282

Maeda, K., Nozawa, T., Sahu, D. K., et al. 2013, ApJ, 776, 5

Margutti, R., Milisavljevic, D., Soderberg, A. M., et al. 2014, ApJ, 780, 21

Martin, J. C., Hambsch, F.-J., Margutti, R., et al. 2015, AJ, 149,

Mauerhan, J. C., Smith, N., Filippenko, A. V., et al. 2013, MNRAS, 430, 1801

Mauerhan, J., Williams, G. G., Smith, N., et al. 2014, MNRAS, 442, 1166

Maza, J., Hamuy, M., Antezana, R., et al. 2009, Central Bureau Electronic Telegrams, 1928, 1

Moriya, T., Tominaga, N., Tanaka, M., et al. 2010, ApJ, 719, 1445

Moriya, T. J. 2014a, A\&A, 564, AA83

Moriya, T. J. 2014b, arXiv:1402.2519

Moriya, T. J., Maeda, K., Taddia, F., et al. 2014, MNRAS, 439, 2917

Moriya, T. J., Blinnikov, S. I., Tominaga, N., et al. 2013a, MNRAS, 428, 1020
Moriya, T. J., Maeda, K., Taddia, F., et al. 2013b, MNRAS, 435, 1520

Murase, K., Thompson, T. A., \& Ofek, E. O. 2014, MNRAS, 440, 2528

Murase, K., Thompson, T. A., Lacki, B. C., \& Beacom, J. F. 2011, Phys. Rev. D, 84, 043003

Nadezhin, D. K. 1980, Ap\&SS, 69, 115

Ofek, E. O., Lin, L., Kouveliotou, C., et al. 2013a, ApJ, 768, 47

Ofek, E. O., Sullivan, M., Cenko, S. B., et al. 2013b, Nature, 494, 65

Ofek, E. O., Zoglauer, A., Boggs, S. E., et al. 2014, ApJ, 781, 42

Ouyed, R., Koning, N., \& Leahy, D. 2013, Research in Astronomy and Astrophysics, 13, 1463

Pastorello, A., Cappellaro, E., Inserra, C., et al. 2013, ApJ, 767, 1

Potashov, M., Blinnikov, S., Baklanov, P., \& Dolgov, A. 2013, MNRAS, 431, L98

Prieto, J. L., Brimacombe, J., Drake, A. J., \& Howerton, S. 2013, ApJL, 763, LL27

Quataert, E., \& Shiode, J. 2012, MNRAS, 423, L92

Smith, N. 2014, ARA\&A, 52, 487

Smith, N., Mauerhan, J. C., \& Prieto, J. L. 2014, MNRAS, 438, 1191

Smith, N., Mauerhan, J. C., Kasliwal, M. M., \& Burgasser, A. J. 2013, MNRAS, 434, 2721

Smith, N., Miller, A., Li, W., et al. 2010, AJ, 139, 1451

Soker, N., \& Kashi, A. 2013, ApJL, 764, LL6

Svirski, G., Nakar, E., \& Sari, R. 2012, ApJ, 759, 108

Tanaka, M., Moriya, T. J., Yoshida, N., \& Nomoto, K. 2012, MNRAS, 422, 2675

Tsebrenko, D., \& Soker, N. 2013, ApJL, 777, LL35

Van Dyk, S. D., Peng, C. Y., King, J. Y., et al. 2000, PASP, 112 , 1532

van Marle, A. J., Smith, N., Owocki, S. P., \& van Veelen, B.

2010, MNRAS, 407, 2305

Weaver, T. A. 1976, ApJS, 32, 233 